DOI:

10.1038/nrm2448

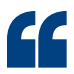

.... a third

model that

involves

the rapid

partitioning of

Golgi enzymes

and trans-

membrane

cargo...

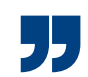

MEMBRANE TRAFFICKING

\title{
All cisternae are not created equal
}

There are two main models for intraGolgi transport of cargo proteins: the vesicular transport model and the cisternal maturation (or progression) model, the latter being the most widely accepted. New data reported by Jennifer Lippincott-Schwartz and colleagues call these models into question and suggest a third model that involves the rapid partitioning of Golgi enzymes and transmembrane cargo between two lipid phases, combined with a rapid exchange among cisternae.

A prediction from the cisternal maturation model is that there is a lag time before cargo leaves the Golgi and that its exit kinetics are linear. To test this prediction, the authors selectively photobleached all fluorescently labelled cargo (large soluble, small soluble and transmembrane) outside the Golgi and then quantified the rate at which cargo exited. All three types of cargo exited the Golgi with exponential rather than linear kinetics. Next, the authors photobleached all fluorescent cargo in the Golgi, after which labelled cargo was allowed to enter the Golgi for 5 minutes, and all fluorescence outside the Golgi was photobleached. They found that the pulse-labelled cargo exited the Golgi with exponential kinetics without a lag time. This suggests that all cargo is equally likely to leave the Golgi from all cisternae.

Lippincott-Schwartz and coworkers tagged a Golgi enzyme with one fluorescent marker and a cargo protein with another, and then selectively photobleached the fluorescent cargo and quantified the distribution of newly arrived cargo to Golgi cisternae using two-colour imaging. Cargo did not distribute equally into all Golgi compartments - transport intermediates were seen to bud from regions that were enriched in labelled cargo protein ('export domains'),

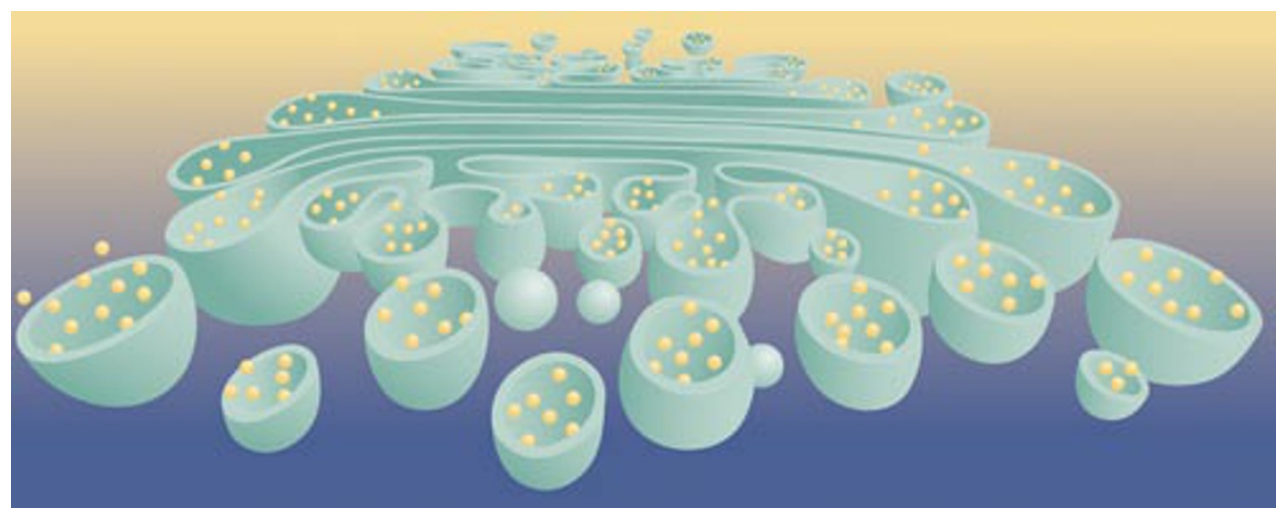

whereas 'processing domains' were enriched in labelled Golgi enzyme. However, the transport rate among cisternae was high, which is consistent with the notion that cisternae are continuous. The authors therefore suggested a new 'membrane partitioning' model in which cargo moves between two different Golgi membrane locations.

The authors developed this model on the basis of reported observations regarding the lipid composition of the Golgi and the differential affinity of resident enzymes and cargo proteins for different lipid compositions. Simulation of the membrane partitioning model accounted for some key characteristics of Golgi transport and organization, including a cis-to-trans gradient of glycerophospholipids and sphingolipids, the distribution of resident proteins in the Golgi stack and exponential cargo release kinetics. The model can explain some apparent discrepancies in the literature, while it also raises new questions; for example, what are the transport vehicle(s) (that is, vesicles or tubules) that Golgi cargo uses in this model?

Arianne Heinrichs

ORIGINAL RESEARCH PAPER Patterson, G. H. et al. Transport through the Golgi apparatus by rapid partitioning within a two-phase membrane system. Cell 133, 1055-1067 (2008) FURTHER READING De Matteis, M. A. \& Luini, A. Exiting the Golgi complex. Nature Rev. Mol. Cell Biol. 9, 273-284 (2008) 